\title{
Oxidation of tunnel barrier metals in magnetic tunnel junctions
}

\author{
J. Joshua Yang, Peter F. Ladwig, ${ }^{\text {a) }}$ Ying Yang, Chengxiang Ji, and Y. Austin Chang ${ }^{\text {b) }}$ \\ Department of Materials Science and Materials Science Program, University of Wisconsin-Madison, Madison, \\ Wisconsin 53706
}

Feng X. Liu, Bharat B. Pant, and Allan E. Schultz

Recording Head Operations, Seagate Technology, Bloomington, Minnesota 55431

(Presented on 10 November 2004; published online 5 May 2005)

\begin{abstract}
The oxidation of an ultrathin metal layer $(<1 \mathrm{~nm})$ to form an oxide tunnel barrier is of critical importance for the fabrication of magnetic tunnel junctions (MTJs) with low product of resistance and area $(R \times A)$. Nonuniform and excessive or insufficient oxidation will occur by using conventional plasma, air, or $\mathrm{O}_{2}$ and noble gas mixtures as oxidation methods. An oxidation method was investigated to oxidize only an ultrathin layer of metal (such as Y) without oxidizing adjacent ferromagnetic thin film layers. We have now demonstrated that a gas mixture of $\mathrm{H}_{2} \mathrm{O} / \mathrm{H}_{2}$ with a fixed chemical potential of oxygen determined by the relative amounts of the two gases can oxidize Y and Ta thin layers while simultaneously keeping a Co ferromagnetic layer completely unoxidized. This universal method can be used to preferentially oxidize a host of other metals with high tendency to form oxides, such as $\mathrm{Zr}, \mathrm{Hf}, \mathrm{Nb}$, rare earth metals, etc. and may allow us to access the feasible lower limit of barrier thickness in MTJs. (C) 2005 American Institute of Physics.

[DOI: 10.1063/1.1854011]
\end{abstract}

\section{INTRODUCTION}

The tunneling magnetoresistive (TMR) read head is one device that has been investigated as a candidate for replacing traditional GMR heads as recording densities move beyond 100 Gbit/in. ${ }^{2}$. A magnetic tunnel junction (MTJ) consists of a tunnel barrier layer made from a nonmagnetic insulating material sandwiched between two ferromagnetic (FM) layers. The nonmagnetic insulating material is usually oxide, which is formed by oxidizing the predeposited tunnel barrier metal on top of the FM layer. It was reported that the product of junction resistance and area $(R \times A)$ for magnetic recording read heads applications might have to be in the order of few $\Omega \mu \mathrm{m}^{2}$. To fulfill this low resistance requirement for MTJs with oxide barriers, the precursor metal should be ultrathin $(<1 \mathrm{~nm}) .{ }^{1,2}$ Unfortunately, it is extremely difficult, if not impossible, to completely oxidize such a thin tunnel barrier metal in the MTJ without at least partially oxidizing the neighboring ferromagnetic layers using conventional oxidation methods. In the production of a MTJ it is important to use an insulating tunnel barrier layer that is neither overoxidized nor underoxidized, as both conditions will affect the performance of the device. ${ }^{3}$ In this paper, a method of oxidizing only the tunnel barrier precursor metal was investigated. We have now demonstrated that a gas mixture of $\mathrm{H}_{2} \mathrm{O} / \mathrm{H}_{2}$ with a fixed chemical potential of oxygen determined by the relative amounts of the two gases can oxidize $\mathrm{Y}$ and Ta thin layers while simultaneously leaving a Co ferromagnetic layer completely unoxidized.

\footnotetext{
${ }^{a)}$ Present address: Advanced Process Development, Hutchinson Technology, Hutchinson, MN.

b) Author to whom correspondence should be addressed; electronic mail: chang@engr.wisc.edu
}

\section{EXPERIMENT}

Multilayer films of seed layer metal $(100 \mathrm{~nm}) / \mathrm{M} / \mathrm{cap}$ layer metal $(3 \mathrm{~nm})$ were deposited on $\mathrm{Si}$ substrates with the native oxide layer by dc magnetron sputtering, where $M$ stands for different tunnel barrier metals, i.e., $\mathrm{Y}$ and Ta. In some samples, a $2 \mathrm{~nm}$ Co layer was deposited in between $100 \mathrm{~nm}$ seed layer metal and tunnel barrier precursor metal layer to test if the underlying ferromagnetic layer was oxidized during the tunnel barrier metal oxidation process. Prior to the deposition of $3 \mathrm{~nm}$ cap layer, the tunnel barrier metals were oxidized in situ with gas mixtures containing $\mathrm{H}_{2} \mathrm{O} / \mathrm{H}_{2}$ at varying total pressures. The ratios of $\mathrm{H}_{2} \mathrm{O} / \mathrm{H}_{2}$ adopted were $2 E-4$. During the oxidation process, the samples could be heated up by the built-in resistor heater in the sample holder. The chemical states of the metal elements were characterized by PHI 5400 x-ray photoelectron spectroscopy (XPS).

\section{RESULTS AND DISCUSSION}

Plasma oxidation or natural oxidation (using air, $\mathrm{O}_{2}$, or mixture of these with noble gases) of tunnel barrier precursor metals is kinetically controlled, since the oxidation time must be precisely known in order not to overoxidize/underoxidize the tunnel barrier. In contrast, the selective oxidation method is thermodynamically controlled. The main idea central to this method is that the tunnel barrier precursor metal can be oxidized but not the adjacent FM layers with a precise control of the chemical potential of $\mathrm{O}_{2}$ within a window where the FM metal (e.g., Co) and tunnel barrier oxide (e.g., $\mathrm{Y}_{2} \mathrm{O}_{3}$ ) coexist in two phase equilibrium. The defined chemical potential of $\mathrm{O}_{2}$ for this purpose is very low and is not attainable by introducing a known amount of $\mathrm{O}_{2}$ into a high purity carrier gas such as Ar in practical applications. However, it is 


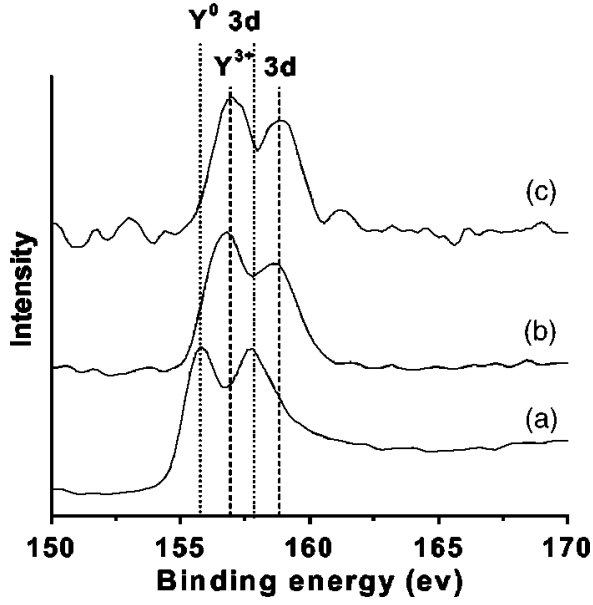

FIG. 1. XPS spectra of Y $3 d$ from Y layers. (a) $0.8 \mathrm{~nm}$ pure $\mathrm{Y}$ deposited at room temperature. (b) $0.8 \mathrm{~nm} \mathrm{Y}$ layer subjected by 0.5 Torr $\mathrm{O}_{2}$ at room temperature for $5 \mathrm{~min}$. (c) $0.8 \mathrm{~nm}$ Y layer subjected by $100 \mathrm{Torr} \mathrm{H}_{2} \mathrm{O} / \mathrm{H}_{2}$ at room temperature for $40 \mathrm{~min}$.

possible to reach the low $\mathrm{O}_{2}$ chemical potential by controlling the ratio of a certain kind of gas mixture, such as $\mathrm{H}_{2} \mathrm{O} / \mathrm{H}_{2}$. For example, the chemical potential of $\mathrm{O}_{2}$ determined by the $\mathrm{H}_{2} \mathrm{O}$ and $\mathrm{H}_{2}$ gas mixture can be calculated by the following reaction, in which $\mathrm{K}$ is equilibrium constant and $P_{\mathrm{O}_{2}}$ is the oxygen partial pressure associated with the oxygen chemical potential.

$$
\mathrm{H}_{2}+\frac{1}{2} \mathrm{O}_{2}=\mathrm{H}_{2} \mathrm{O}, \quad P_{\mathrm{O}_{2}}=\left[\frac{P_{\mathrm{H}_{2} \mathrm{O}}}{P_{\mathrm{H}_{2}}} \frac{1}{K}\right]^{2} \text {. }
$$

With thermodynamic data for the FM metal, tunnel barrier precursor metal and oxygen ternary system, a proper ratio range of gas mixture $\left(P_{\mathrm{H}_{2} \mathrm{O}} / P_{\mathrm{H}_{2}}\right)$ can be calculated to oxidize the tunnel barrier metal but not the FM metal. Details of this concept will be published elsewhere.

Figure 1 shows XPS results from Y layers subjected to thermodynamically controlled oxidation in $\mathrm{H}_{2} \mathrm{O} / \mathrm{H}_{2}$, as well as results from pure $\mathrm{Y}\left(\mathrm{Y}^{\circ}\right)$ and $\mathrm{Y}$ oxidized in pure $\mathrm{O}_{2}\left(\mathrm{Y}^{3+}\right)$. The thermodynamically controlled oxidation samples are aligned with that from the sample oxidized by oxygen, indicating that the gas mixture did oxidize the $\mathrm{Y}$ layer. The oxidation of $0.7 \mathrm{~nm}$ Ta thin films by $\mathrm{H}_{2} \mathrm{O} / \mathrm{H}_{2}$ is shown in Fig. 2 . It is worth noting that there is a slight shoulder in the curve Fig. 2(c) corresponding to $\mathrm{Ta}^{\circ} 4 f$, which suggests that $0.7 \mathrm{~nm}$ Ta was not completely oxidized under that oxidation condition. This illustrates the point that although the reaction is thermodynamically controlled, there are kinetic factors, which come into play until the equilibrium condition is established. Obviously, the oxidation reaction kinetics is quite different for different tunnel barrier metals. This kinetic difference can be related to differences in metal work functions. Lower work functions result in higher oxygen uptake, increase the rate of dissociation of oxygen molecules at the surface, and lower the barrier for oxygen penetration into the surface. ${ }^{5}$ It can be expected that metals with low work functions, such as $\mathrm{Zr}$, Hf, Gd, Ce, Ba, etc. would be easily oxidized by the gas mixture.

In order to meet the low resistance requirement for MTJs, the tunnel barrier metals need to be ultrathin

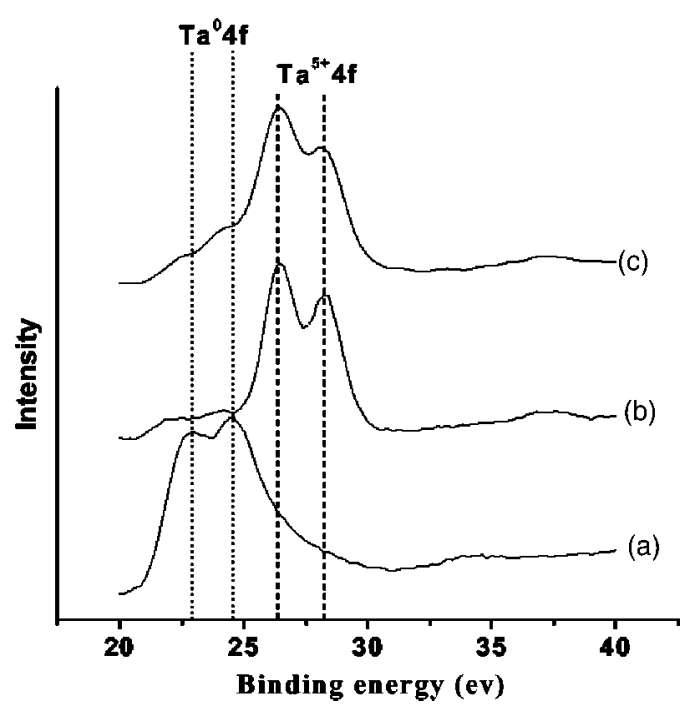

FIG. 2. XPS spectra of Ta $4 f$ from Ta layers. (a) $0.7 \mathrm{~nm}$ pure Ta deposited at room temperature. (b) $0.7 \mathrm{~nm}$ Ta layer subjected by air at room temperature for $60 \mathrm{~min}$. (c) $0.7 \mathrm{~nm}$ Ta layer subjected by $500 \mathrm{Torr} \mathrm{H}_{2} \mathrm{O} / \mathrm{H}_{2}$ at elevated temperature for $60 \mathrm{~min}$.

$(<1 \mathrm{~nm})$. However, if the thickness of tunnel barrier metal is less than $1 \mathrm{~nm}$, then the underlying FM layer is very vulnerable to oxygen using the conventional plasma or natural oxidation methods. A Co layer covered by $0.8 \mathrm{~nm}$ Y layer was exposed to 0.5 Torr $\mathrm{O}_{2}$ at room temperature for just $5 \mathrm{~min}$ and there are distinct $2 p$ peaks from $\mathrm{Co}^{2+}$ as illustrated in Fig. 3(b). However, as shown in Fig. 3(a), the same structure subjected to 500 Torr of the $\mathrm{H}_{2} \mathrm{O} / \mathrm{H}_{2}$ gas mixture at $200{ }^{\circ} \mathrm{C}$ for $60 \mathrm{~min}$ does not have oxidized Co peaks, although the $0.8 \mathrm{~nm}$ top Y layer was completely oxidized.

\section{CONCLUSIONS}

It has been shown that gas mixtures of $\mathrm{H}_{2} \mathrm{O} / \mathrm{H}_{2}$ with proper ratios could be used to control the oxygen chemical potential and thus only oxidize tunnel barrier metals, such Y,

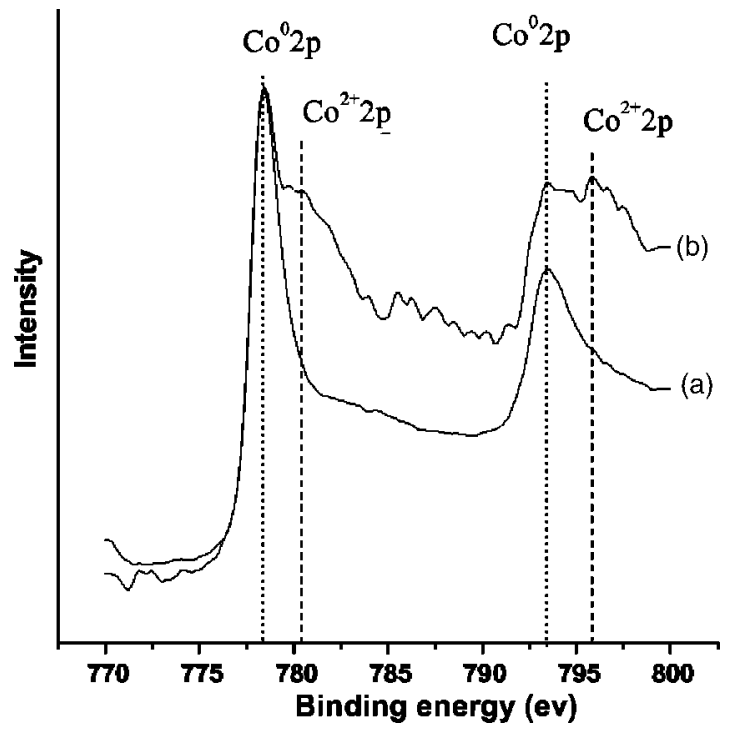

FIG. 3. XPS spectra of Co $2 p$ from underlying Co layer after the $0.8 \mathrm{~nm} \mathrm{Y}$ layers (on top of Co) were oxidized by (a) 500 Torr $\mathrm{H}_{2} \mathrm{O} / \mathrm{H}_{2}$ at elevated temperature for $60 \mathrm{~min}$ and (b) 0.5 Torr $\mathrm{O}_{2}$ at room temperature for $5 \mathrm{~min}$. 
Ta, and so on. The underlying FM layer is very vulnerable to oxygen using the conventional plasma or natural oxidation methods when the tunnel barrier precursor metal is ultrathin. This oxidation method may allow us to access the lower limit of barrier thickness in MTJs.

\section{ACKNOWLEDGMENTS}

This work was supported by a grant from the Division of Materials Science, Office of Basic Energy Research of De- partment of Energy under Grant No. DE-FG02-99ER45777, Seagate Technology, and the Wisconsin Distinguished Professorship.

${ }^{1}$ J. Wang, P. P. Freitas, and E. Snoeck, Appl. Phys. Lett. 79, 4553 (2001). ${ }^{2}$ B. G. Park, T. D. Lee, T. H. Lee, C. G. Kim, and C. O. Kim, J. Appl. Phys. 93, 6423 (2003).

${ }^{3}$ J. S. Moodera, E. F. Gallagher, K. Robinson, and J. Nowak, Appl. Phys. Lett. 70, 3050 (1997).

${ }^{4}$ P. F. Ladwig, J. J. Yang, Y. Yang, F. Liu, B. B. Pant, A. E. Schultz, and Y. A. Chang (unpublished).

${ }^{5}$ N. A. Braaten, J. K. Grepstad, S. Raaen, and S. L. Qiu, Surf. Sci. 250, 51 (1991). 